\title{
A Performance Comparison of Systematic Polar Codes and Non-systematic Polar Codes
}

\author{
Ming Ye and Hui Li ${ }^{*}$ \\ School of Information Science and Technology, Hainan University, Haikou 570228, China \\ ${ }^{*}$ Corresponding author
}

\begin{abstract}
Polar coding is a code construction method that can achieve the capacity of symmetric binary-input discrete memoryless channels (B-DMC). Polar codes in standard form are non-systematic polar codes (NSPCs). The codes are susceptible to error propagation under successive cancellation (SC) decoding while systematic polar codes (SPCs) are more robust against error propagation. SPCs may be considered as a generalization of NSPCs. Here, we demonstrate the performance advantages of SPCs over NSPCs under SC decoding.
\end{abstract}

Keywords-polar codes; systematic polar codes; non-systematic polar codes; successive cancellation decoding

\section{INTRODUCTION}

Polar coding is a code construction method that can achieve the symmetric capacity $I(W)$ of binary-input discrete memoryless channels [1], and polar codes in standard form are NSPCs. The codes originally introduced in [1] are a class of non-systematic linear block codes. Any linear code can be turned into a systematic code. Hence, NSPCs can also be encoded systematically. However, it is unclear if this can be done while retaining the low-complexity nature of polar coding [2]. It is also not clear if polar codes encoded systematically have advantages over those encoded non-systematically in terms of performance. The encoding methods of NSPCs and SPCs are introduced. The encoding method of SPCs preserves the low-complexity nature while guaranteeing the same frame error rate (FER) [2]. The method is also applicable to turbo-style receiver designs and Reed-Muller codes. Simulation results show that SPCs have better performance of bit error rate (BER) than NSPCs while they have same FER performance.

\section{NON-SYSTEMATIC POLAR CODES}

Let code length $\mathrm{N}=2^{\mathrm{n}}, \mathrm{n}=1,2, \ldots$, and information block length $\mathrm{K}$,then code rate $\mathrm{R}=\mathrm{K} / \mathrm{N}$. Given a B-DCM $W: \chi \rightarrow \gamma$, where $\chi=\{0,1\}$ and $\gamma$ denote the input and output alphabets respectively and its transition probability is denoted by $\mathrm{W}(\mathrm{y} \mid \mathrm{x})$.We write $u_{i}^{j}$ to denote the vector $\left(u_{i}, u_{i+1}, \cdots, u_{j}\right) . \mathrm{N}$ subchannels $W_{N}^{(i)}: \chi \rightarrow \gamma^{N} \times \chi^{i-1}, 1 \leq i \leq N$ are got by performing channel combining and splitting operations on $\mathrm{N}$ independent uses of $\mathrm{W}$ and

$$
W_{N}^{(i)}\left(y_{1}^{N}, u_{1}^{i-1} \mid u_{i}\right)=\sum_{u_{i+1}^{N} \in \chi^{N-i}} \frac{1}{2^{N-1}} W_{N}\left(y_{1}^{N} \mid u_{1}^{N}\right)
$$

When the code length $\mathrm{N}$ is sufficiently large, the fraction of clean channels turns to be close to the symmetric capacity $I(W)$ and others are completely unreliable. In fact, polar codes of dimension $\mathrm{K}$ are constructed by assigning $\mathrm{K}$ information bits on the $\mathrm{K}$ most reliable subchannels $W_{N}^{(i)}$. Information bits set $I$ is the set of those channels, i.e., $i \in I,|I|=K$. The frozen bits set is denoted by the complementary set of I,i.e., I ${ }^{\mathrm{c}}$. The reliability of $W_{N}^{(i)}$ can be estimated by using Bhattaharyya parameter [1] for binary erasure channel (BEC). While the reliability can also be computed by density evolution or Gaussian approximation method for other channels [3] [4]. For a given N, polar codes are encoded in the same manner, namely

$$
x_{1}^{N}=u_{1}^{N} G_{N}
$$

where $u_{1}^{N}$ which contains information bits $u_{I}$ and frozen bits $u_{I^{c}}$ denotes the source block, and $x_{1}^{N}$ is the code block. $\mathrm{G}_{\mathrm{N}}$ is the generator matrix of order $\mathrm{N}$, defined as

$$
G_{N}=B_{N} F_{2}^{\otimes n}
$$

Where $B_{N}$ is a bit-reversal permutation matrix, $F_{2}=\left[\begin{array}{ll}1 & 0 \\ 1 & 1\end{array}\right]$, and $\otimes n$ is the n-th Kronecker power. Since the information bits are assigned to the side of source block $u_{I}$ not the side of code block $x_{1}^{N}$, polar codes with this scheme are NSPCs [5].

As mentioned in [1], SC decoder is considered to generate its decision $\hat{u}_{1}^{N}$ by computing

$$
\hat{u}_{i}= \begin{cases}u_{i}, & i \in I^{c} \\ h_{i}\left(y_{1}^{N}, \hat{u}_{1}^{i-1}\right), & i \in I\end{cases}
$$

in the order $\mathrm{i}$ from 1 to $\mathrm{N}$, where $h_{i}: \gamma^{N} \times \chi^{i-1} \rightarrow \chi, i \in I$ are decision functions defined as 


$$
h_{i}\left(y_{1}^{N}, u_{1}^{i-1}\right)= \begin{cases}0, & \frac{w_{N}^{(i)}\left(y_{1}^{N}, \hat{u}_{1}^{i-1} \mid 0\right)}{w_{N}^{(i)}\left(y_{1}^{N}, \hat{u}_{1}^{i-1} \mid 1\right)} \geq 1 \\ 1, & \text { otherwise }\end{cases}
$$

\section{Systematic Polar CoDES}

After splitting the source word into two parts $u=\left(u_{A}, u_{A^{c}}\right)$ for some $A \subset\{1, \ldots, N\}$, a class of codes are defined using (2). $u_{A}=\left(u_{i}: i \in A\right)$ consists of information bits that are free to change in each round of transmission while the frozen bits are made known to the decoder. (2) is revised as

$$
x=u_{A} G_{A}+u_{A^{c}} G_{A^{c}}
$$

where $G_{A}$ and $G_{A^{c}}$ are the submatrices of $G$ consisting of rows with indices in $\mathrm{A}_{\text {and }} \mathrm{A}_{\mathrm{c}}$, respectively. This mapping represents a non-systematic encoder $u_{A} \mapsto x=u_{A} G_{A}+c$ where $c \triangleq u_{A^{c}} G_{A^{c}}$ is a fixed vector.

In order to consider various possible systematic encoders for the code, as specified by a non-systematic encoder as in (6), let us split the codeword into two parts $x=\left(x_{B}+x_{B^{c}}\right)$. B is an arbitrary subset of $\{1, \ldots, N\}$. (6) is revised as

$$
\begin{gathered}
x_{B}=u_{A} G_{A B}+u_{A^{c}} G_{A^{c} B} \\
x_{B^{c}}=u_{A} G_{A B^{c}}+u_{A^{c}} G_{A^{c} B^{c}}
\end{gathered}
$$

Where $G_{A B}$ represents the submatrix of $G$ consisting of the array of elements $\left(G_{i, j}\right), i \in A, j \in B$. For systematic encoding, $\mathrm{x}_{\mathrm{B}}$ plays the role $\mathrm{u}_{\mathrm{A}}$ played in non-systematic encoding as the data carrier, while $u_{A^{c}}$ is fixed as before. As mentioned in [2], for a given non-systematic encoder with parameter $\left(A, u_{A^{c}}\right)$, a systematic encoder with parameter $\left(B, u_{A^{c}}\right)$ exists if equations (7) and (8) establish a one-to-one correspondence between the sets of possible values of $\mathrm{u}_{\mathrm{A}}$ and $\mathrm{x}_{\mathrm{B}}$. Similarly, when A and B have the same number of elements and $\mathrm{G}_{\mathrm{AB}}$ is an invertible matrix, polar codes with parameter $\left(A, u_{A^{c}}\right)$ can be turned into systematic polar codes with parameter $\left(B, u_{A^{c}}\right)$ [2]. In this case, after computing

$$
u_{A}=\left(x_{B}-u_{A^{c}} G_{A^{c} B}\right)\left(G_{A B}\right)^{-1}
$$

and then inserting $\mathrm{u}_{\mathrm{A}}$ into (8), a systematic encoder with parameter can implement the mapping $x_{B} \mapsto X=\left(x_{B}, x_{B^{c}}\right)$.

\section{Performance Analysis}

In this part, sufficient evidence is provided to conclude that SPCs are better than NSPCs. Simulations reported here were conducted using polar codes with different code lengths and code rates over an additive white Gaussian noise (AWGN) channel. We employed the same SC decoder [1] for both SPCs and NSPCs. The modulation was binary phase shift keying (BPSK). For non-systematic encoding, the decoder stopped after producing an estimate $\hat{u}$ of $\mathrm{u}$ and $\hat{u}_{A}$ as output; for NSPCs, BER and FER statistics were compiled by computing $\mathrm{u}_{\mathrm{A}}$ and
$\hat{u}_{A}$. For systematic encoding, the decoder computed an estimate $\hat{x}=\hat{u} G_{N}$ of $\mathrm{x}$, and put out $\hat{x}_{A}$.

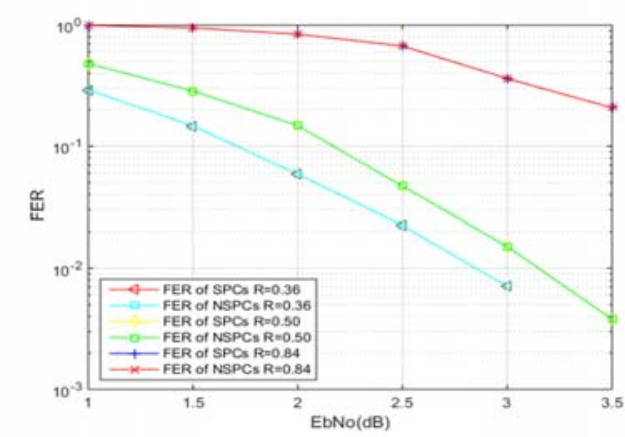

FIGURE I. FER OF SPCS AND NSPCS WITH DIFFERENT CODE RATES,CODE LENGTH N=256

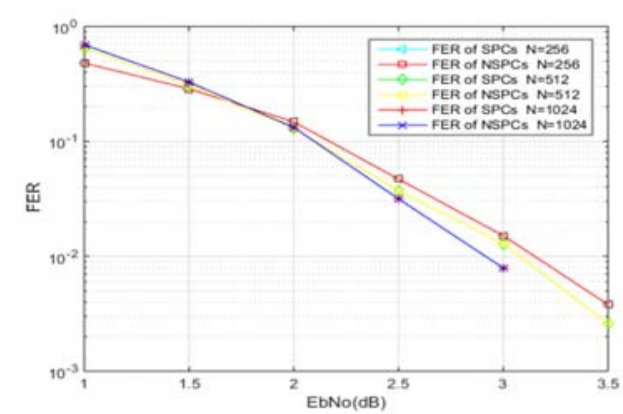

FIGURE II. FER OF SPCS AND NSPCS WITH DIFFERENT CODE LENGTHS,CODE RATE $\mathrm{R}=1 / 2$

Fig. 1 gives FER performance of SPCs and NSPCs with various code rates. Through observing Fig.1, it is found that SPCs and NSPCs have the same performance of FER. However, Fig.1 does not provide sufficient evidence to conclude that they have the same FER performance. To that end, we consider polar codes with different code lengths as given in Fig.2. The simulation results show again that performance of FER of SPCs and NSPCs are coincident. Fig. 3 shows the simulation results of BER of SPCs and NSPCs. The performance improvement of SPCs is especially pronounced at low $\mathrm{E}_{\mathrm{b}} / \mathrm{N}_{\mathrm{o}}$ values.

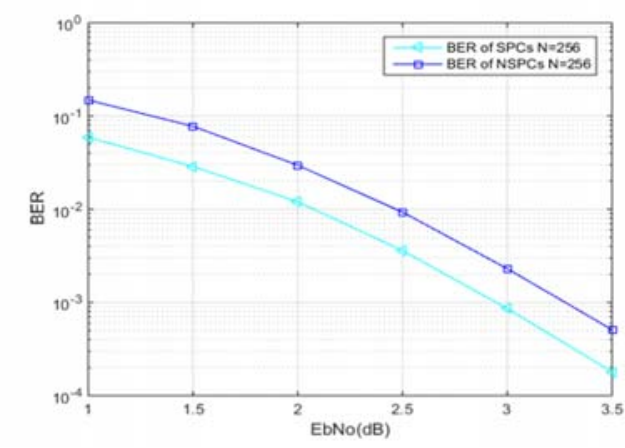

FIGURE III. BER OF SPCS AND NSPCS WITH CODE LENGTH $\mathrm{N}=256$,CODE RATE $\mathrm{R}=1 / 2$ 


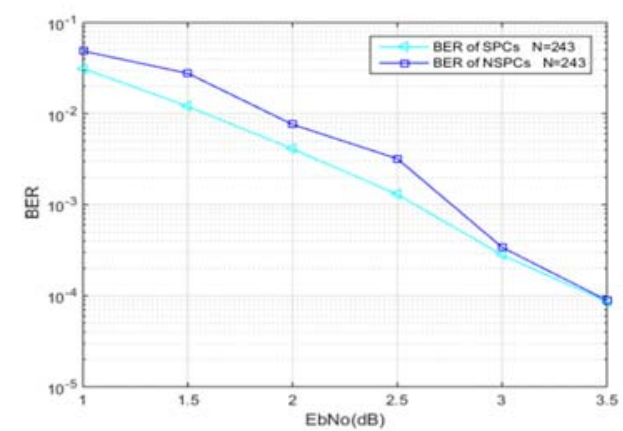

FIGURE IV. BER OF SPCS AND NSPCS WITH CODE LENGTH $\mathrm{N}=243$,CODE RATE $\mathrm{R}=1 / 2$

The simulation results of BER performance of SPCs and NSPCs based on $3 \times 3$ kernel matrix [6] are also given in Fig. 4 . The results also indicates that SPCs have better BER performance compared with NSPCs. Here, the list size of successive cancellation list decoder is set to 32. It is surprising, however, that any decoding errors in $\hat{u}$ would be amplified in the re-encoding step $\hat{x}=\hat{u} G_{N}$. Paradoxically, the simulation results show that this is not the case. This issue is a subject for further study.

\section{CONCLUSION}

SPCs are more robust against error propagation than NSPCs since the information bits in systematic encoding are directly observed through the channel. The simulation results demonstrate that SPCs have advantage over NSPCs in BER performance while they have the same FER performance.

\section{ACKNOWLEDGMENT}

This work is supported by the National Natural Science Foundation of China (No. 61661018), International Cooperation Special of Ministry of Science and Technology (No. 2015DFR10510), Hainan Key R\&D Plan High-tech Project (No. ZDYF2016010), Hainan Natural Science Foundation (No. 20166210) and Key Project of Scientific Research of Higher Institution of Hainan Provincial Education Department (No. Hnky2016ZD-5).

\section{REFERENCES}

[1] E. Arikan, "Channel polarization: A method for constructing capacity achieving codes for symmetric binary-input memoryless channels," IEEE Trans. Inf. Theory, vol. 55, no.7, pp. 3051-3073, Jul.2009.

[2] E. Arikan, "Systematic polar codes," IEEE Communications Letters, vol. 15,no.8,pp.860-862,August 2011.

[3] T. Richardson, R. Urbanke, Modern Coding Theory, Cambridge, UK: Cambridge University Press, 2008.

[4] R. Mori, T. Tanaka, "Performance of polar codes with the construction using density evolution,” IEEE Commun. Lett., vol.56, no. 12, pp. 6253-6264, 2010.

[5] Z. Liu, K. Chen, K. Niu, Z. He, "Distance spectrum analysis of polar codes," IEEE Wireless Communications and Networking Conference, pp. 490-495, 2014.

[6] Z. Liu, K. Niu, C. Dong, "Performance analysis of polar codes based on $3 x 3$ kernel matrix," IEEE International Conference on communications and Networking in China,pp.382-386,2015. 